\title{
Assessment of motion-induced fluidization of dense pyroclastic gravity currents
}

\author{
Piero Salatino \\ Dipartimento di Ingegneria Chimica, Università degli Studi di Napoli «Federico II», Napoli, Italy
}

\begin{abstract}
The paper addresses some fundamental aspects of the dynamics of dense granular flows down inclines relevant to pyroclastic density currents. A simple mechanistic framework is presented to analyze the dynamics of the frontal zone, with a focus on the establishment of conditions that promote air entrainment at the head of the current and motion-induced self-fluidization of the flow. The one-dimensional momentum balance on the current along the incline is considered under the hypothesis of strongly turbulent flow and pseudo-homogeneous behaviour of the two-phase gas-solid flow. Departures from one-dimensional flow in the frontal region are also analyzed and provide the key to the assessment of air cross-flow and fluidization of the solids in the head of the current. The conditions for the establishment of steady motion of pyroclastic flows down an incline, in either the fluidized or «dry» granular states, are examined.
\end{abstract}

Key words pyroclastic flow - fluidization - gravity current

\section{Introduction}

Considerable progress has been made over the last decade in mechanistic understanding of the establishment and flow of pyroclastic gravity currents (Druitt, 1998; Burgisser and Bergantz, 2002; Neri et al., 2002b). In particular, the role of gases - and of their interaction with pyroclastic solids - on the establishment of dense versus dilute suspension currents as a result of explosive volcanism has been highlighted. On one side, dense pyroclastic gravity currents result from limited amount of gas in the eruptive mixture leaving the crater, insufficient to produce a fully convective buoyant column. On the other, the amount of gas entrained by the current in the at-

Mailing address: Prof. Piero Salatino, Dipartimento di Ingegneria Chimica, Università degli Studi di Napoli «Federico II», P.le V. Tecchio 80, 80125 Napoli, Italy; e-mail: salatino@unina.it mosphere is largely responsible for its mobility and hazard.

The role of fluidization in the emplacement of dense pyroclastic flows has long been recognized (Spark, 1976; Wilson, 1980, 1984; Marzocchella et al., 1998; Roche et al., 2002; Druitt et al., 2004; Gravina et al., 2004). Notwithstanding, quantitative assessment of fluidization in pyroclastic flows is still extremely poor. Broad uncertainties still characterize fundamental aspects of pyroclastic flows, like: a) the prevailing nature and source of the fluidizing gas, either endogenous or associated with entrainment phenomena; b) the rheology of aerated/fluidized gas-solid two-phase dense flows under high-velocity strongly turbulent conditions; c) the perturbation to the classical phenomenology of fluidization determined by the highly sheared flow conditions; d) the influence of solids polydispersity on fluidization, segregation and flow rheology; e) the expected complexity of bifurcation/dynamical patterns of rapid unsteady granular flows on account of the strong nonlinearity of the governing equations. The current lack of fundamental understanding, despite the extensive published literature on 
rapid granular flows (Savage, 1979; Campbell, 1990), is largely due to the fact that investigations in this field mostly addressed steady flows of granular materials down inclines at velocities far smaller than those typical of pyroclastic flows. Accordingly, these studies completely missed the complex dynamics of the frontal zone, which is bound to be one key to the onset of fluidization phenomena. Moreover, the relative extent of frictional, collisional, streaming and turbulent stresses, which dictates the rheology of the flow, does not even approach conditions relevant to pyroclastic flows.

Due to the intrinsic phenomenological complexity and the lack of experimental results and of adequate theoretical frameworks, modelling of dense pyroclastic currents is still at an embryonic stage and lags far behind the more successful and comprehensive modeling of the dynamics of particle-lean gas-solid suspensions of pyroclastic nature (ash cloud) (Neri et al., 2002a).

The present paper addresses some of the open issues related to quantitative assessment of dense pyroclastic flows from a fundamental perspective. The dynamics of the frontal zone is considered, with an emphasis on the establishment of conditions that promote air entrainment and motion-induced self-fluidization of the flow. An approximate one-dimensional momentum balance on the current steadily flowing along the incline is proposed. It is based on the hypothesis of strongly turbulent flow, of pseudo-homogeneous behaviour of the two-phase gas-solid flow, embodying some of the features that bring about departures from one-dimensional flow. The conditions for the establishemnt of the steady motion-induced fluidization of the pyroclastic flow down an incline are examined.

The proposed mechanistic framework provides the starting point for a broader discussion of research needs and priorities essential to fill the gap of quantitative understanding of unsteady rapid granular flows.

\section{Basic hydrodynamical and rheological features of a dense pyroclastic flow}

A dense pyroclastic flow is a two-phase gravity current moving down an incline (whose slope is $\theta$ ) at a velocity $U$ (fig. 1). The gravity current generates, as it moves, two macroscopic shear layers: a free (i.e. unconfined) shear layer at the top of the gravity current; a confined shear layer at the bottom of the gravity current.

Momentum exchange between the current and the environment takes place via entrainment of the surrounding medium at the upper free shear layer. Momentum exchange between the current and the ground takes place via wall friction at the basal shear layer.

The rheological behaviour of the two-phase gas-solid flow arises from a combination of the following contributions: yield strength due to interparticle friction; stresses due to collisional/kinetic and streaming contributions of the particle phase; viscous/turbulent stresses within

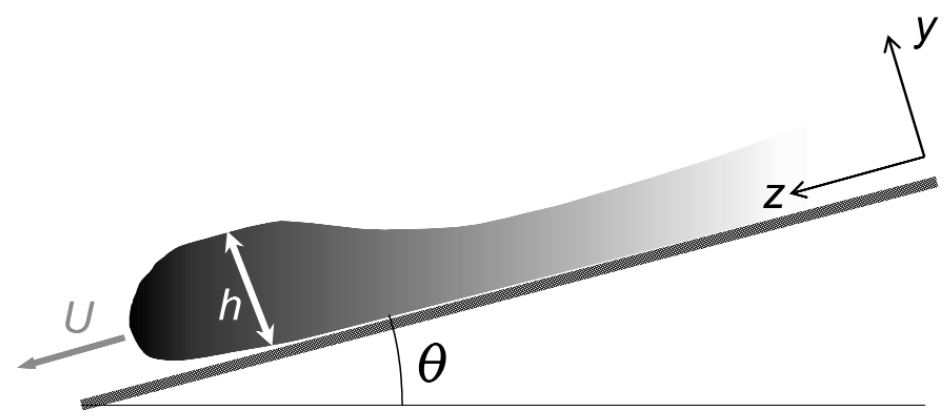

Fig. 1. Outline of a pyroclastic density current. 
the interstitial gas phase; turbulent «Reynolds» stresses at the macroscopic scale.

The first contribution is associated with particle-to-particle stresses of frictional nature. The yield strength is generally expressed as a function of the local normal stress within the particle phase according to the Coulomb Law:

$$
\tau_{z y, f}=\sigma_{y} \cdot \mu=\sigma_{y} \cdot \tan \left(\theta_{0}\right)
$$

where $\mu$ is an internal friction coefficient and $\theta_{\mathrm{o}}$ is the angle of internal friction of the granular solids.

The second contribution is due to momentum transfer via particle-to-particle impact (collisional/kinetic component) or to momentum transfer of convective nature associated with particle velocity fluctuations (streaming component). The shear stress due to this contribution is usually expressed as (Bagnold, 1954; Patton et al., 1987)

$$
\tau_{z y, c}=C_{f} \cdot \rho_{p} d^{2}\left(\frac{d v_{z}}{d y}\right)^{2}=\eta \cdot\left(\frac{d v_{z}}{d y}\right)
$$

where

$$
\eta=C_{f} \cdot \rho_{p} d^{2} \frac{d v_{z}}{d y}=K \cdot\left(\frac{d v_{z}}{d y}\right)
$$

is the effective viscosity of the gas-solid suspension.

The third contribution accounts for deformation of the interstitial gas phase induced by the flow. This is generally neglected on account of the large value of the Bagnold number $\left(B a=\rho_{p} d^{2}\left(d v_{z} / d y\right) / \eta_{g} \gg 1\right)$ for flow conditions of practical interest.

The fourth contribution arises from the onset of turbulence over length scales much larger than the particle scale. It is a momentum transfer of convective nature but, differently from the streaming component, is related to the motion of particle pockets rather than of individual particles.

Altogether, the intrinsic rheological behaviour of the two-phase gas-solid system determined by the combined contribution of the above components is that of a Bingham fluid, non-newtonian, shear-thickening.

If the flow is in the fluidized state, then $\mu=\theta_{\circ}=0$. Accordingly, the yield strength van- ishes. For typical parameters of dense pyroclastic flows $\left(h \cong 1 \mathrm{~m} ; d_{p} \cong 500 \mu \mathrm{m} ; \rho_{m} \cong 100 \mathrm{~kg} / \mathrm{m}^{3}\right.$; $\rho_{p} \cong 1000 \mathrm{~kg} / \mathrm{m}^{3} ; \mathrm{U} \cong 30 \mathrm{~m} / \mathrm{s} ; C_{f}=0.01$ according to Patton et al., 1987) the relevant dimensionless numbers governing the flow of the gravity current take the following values:

Re ynolds: $\quad R e=\frac{3}{2} \frac{\rho_{m} \mathrm{~h}^{2}}{\mathrm{~K}} \cong 10^{7}-10^{8}$

$$
\text { Richardson: } R i=\frac{g \Delta \rho}{\rho_{a}} \frac{h \cos (\theta)}{U^{2}} \cong 1-10
$$

where the expression of the Reynolds number holds for a power-law shear-thickening fluid whose viscosity is given by eq. (2.3) (Levenspiel, 1998). The large values of Richardson number suggest that momentum exchange via air entrainment at the upper free shear layer is negligible (Britter and Linden, 1979). On the other hand, the very large values of Reynolds number indicate that the flow is dominated by inertia.

\section{Momentum balance in the one- dimensional flow approximation}

The hypothesis is made that the flow of the current can be regarded as steady one-dimensional. Furthermore, it is assumed that the current is in the fluidized state and, according to conclusions drawn in the previous section, momentum exchange is dominated by turbulence. The momentum balance equation reads

$$
\rho_{m} g \sin (\theta) h d z=f \frac{\rho_{m} U^{2}}{2} d z
$$

where $z$ is a spatial coordinate along the incline. Equation (3.1) equates the component along the incline of the gravitational force (LHS) to the force exerted on the current by friction at the ground (RHS). Equation (3.1) yields, upon integration

$$
U=\sqrt{\frac{2}{f} g \sin (\theta) h}=\sqrt{\xi h \sin (\theta)} .
$$

Both laboratory data on two-phase fully developed turbulent flows (Wallis, 1969) and field data relative to large scale simulated dry dense snow avalanches (Hopfinger, 1983) suggest that $\xi \approx 1000 \mathrm{~m} / \mathrm{s}^{2}$. 
For small slopes and relevant internal friction the literature on dry dense snow avalanches (Hopfinger, 1983) suggests an alternative form of eq. (3.2)

$$
U=\sqrt{\xi h[\sin (\theta)-\mu \cos (\theta)]} .
$$

\section{Departure from the one-dimensional flow approximation}

The one-dimensional framework within which the momentum balance on the gravity current was developed falls short when the detailed flow structure of the frontal region is analyzed. Phenomenological observation and numerical simulation of dense pyroclastic gravity currents highlight the following features of the flow in the frontal region (fig. 2):

- Flow elements at increasing distance from the ground move faster, possibly overtaking the front. Accordingly 2D and 3D macroscopic flow patterns are established in the vicinity of the front (Valentine and Wohletz, 1989; Todesco et al., 2002). These patterns include fall-out of coarse solids to the ground and vigorous recirculation of the suspension in the head of the current.
- The fall-out of coarse solids compresses the underlying air, which can be either «squeezed out» from the basal region of the front or forced to percolate through it.

- Coupled with the gas flow, finer solids are elutriated through the front contributing to the formation of the upper ash cloud. Fines can also contribute to the momentum exchange between the uprising gas and the coarse solids.

In particular, the second feature deserves attention in the context of the present theoretical development, on account of the possibility that extensive cross-flow of air is driven by overpressures which establish at the base of the current. In turn, cross-flow of air might be large enough to promote fluidization of solids in the head of the current affecting the rheology of the current itself.

\section{An approximate criterion for self- fluidization of a dense granular flow moving down an incline}

It is speculated that, as a consequence of the «average» motion of the front and of the fallout of the solids to the ground (fig. 3), the basal region of the front experiences a value of pres-

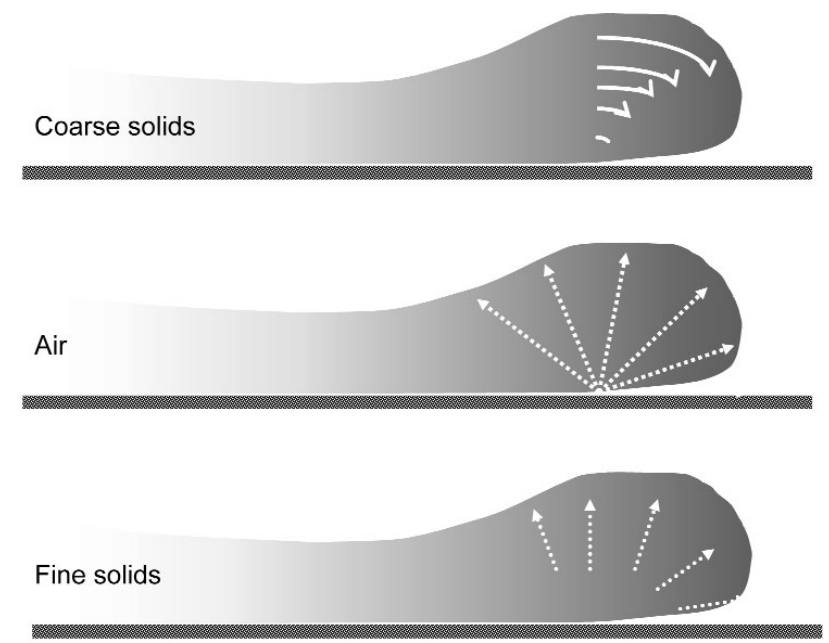

Fig. 2. Solids and gas flow patterns in the head a pyroclastic density current. 


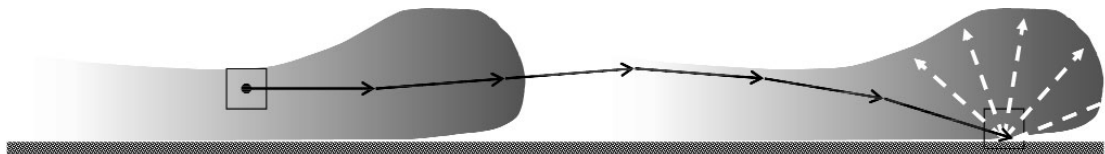

Fig. 3. Assessment of the stagnation pressure in the basal layer of the head of a pyroclastic density current. Solid line: trajectory of a flow element brought to rest as the front is shifted; dashed line: cross-flow of air driven by the stagnation pressure.

sure which is approximately given by

$$
p_{\text {stagnation }} \approx \frac{\rho_{\mathrm{m}} U^{2}}{2}
$$

This value is obtained by application of the Bernoulli equation as the stagnation pressure of a flow element initially moving at the front velocity $U$ as it is brought to rest at the ground. There is general consensus that dynamic pressures in the frontal region of pyroclastic density currents can assume values ranging from a few $\mathrm{kPa}$ up to several tens of $\mathrm{kPa}$ (Valentine, 1998; Esposti Ongaro et al., 2002; Nunziante et al., 2003). When the two-phase nature of the flow is considered, it can be assumed that the stagnation pressure $p_{\text {stagnation }}$ is shared between the interstitial gas phase $p_{g}=\alpha p_{\text {stagnation }}$ and the solid phase $p_{s}=(1-\alpha) p_{\text {stagnation }}$ according to a pressure partition coefficient $\alpha$. In the case $\alpha$ departs significantly from 1 , the assumption of an isotropic particle phase pressure is clearly a gross simplification: it is likely that particle pressure be largely anisotropic under the strongly sheared flow conditions that characterize the motion of dense pyroclastic gravity currents.

The simple idea underlying the self-fluidization criterion is that the overpressure $p_{g}$ in the gas phase associated with the stagnation condition provides the driving force for the flow of air from the basal region of the front across the current. This cross-flow might be large enough to promote fluidization of the current. To this end, the usual condition for fluidization of stationary beds of granular solids is considered: fluidization is established as fluid is supplied at the base of the bed at a relative pressure exceeding the buoyant weight of the bed divided by its cross-sectional area. Of course, one should additionally require that fluid be supplied at the base of the front at a flow rate in excess of that corresponding to incipient fluidization, in order to establish steady cross-flow under self-fluidized conditions: this condition is easily fulfilled if one considers that incipient fluidization velocities of pyroclastic solids typically range between a few $\mathrm{mm} / \mathrm{s}$ and $\mathrm{cm} / \mathrm{s}$ (Gravina et al., 2004), whereas the front of the density current progresses at velocities in the order of tens of $\mathrm{m} / \mathrm{s}$. By combining these concepts, the approximated criterion for the motion-induced self-fluidization of the front is formulated as follows:

«Self-fluidization of the flow occurs if the basal gas pressure $p_{g}$ in the frontal region of the current exceeds the hydrostatic head imposed by the gas-solids suspension».

Or, equivalently

$$
p_{g}=\alpha \frac{\rho_{m} U^{2}}{2}=\alpha \frac{1}{2} \rho_{m} \xi h \sin (\theta) \geq \frac{\rho_{m} g h}{\cos (\theta)} .
$$

Equation (5.2) yields, upon rearrangement and simplification of terms

$$
\frac{\alpha \xi}{2 g} \sin (\theta) \cos (\theta) \geq 1 \text {. }
$$

Equation (5.3) gives the lower limit of the slope $\theta_{c}$ of the incline beyond which self-fluidized flow of the gravity current may onset. The limit value of the slope turns out to be independent of the depth of the current $h$ and of its speed $U$, which cancel out with each other in eq. (5.2). Remarkably, for given flow conditions $\theta_{c}$ depends on the value of the pressure partition coefficient $\alpha$, whose meaning and significance will be addressed later. 
It is worth noting that, according to the present analysis, fulfilment of the criterion given by eq. (5.3) is a necessary but not sufficient condition for the establishment of steady self-fluidized flow of the density current, which is also dependent on the initial conditions of the flow. More specifically, self-fluidization establishes provided that the initial conditions of the flow correspond to a fluidized state. This requirement is met, for instance, if the current results from the fall-out of solids (as in the collapse of an eruptive column).

\section{Implementation of the criterion and discussion of results}

Figure 4 demonstrates the application of the self-fluidization criterion expressed by eq. (5.3). The LHS of eq. (5.3) is plotted versus the slope of the incline $\theta$, the partition coefficient $\alpha$ being treated as a parameter. Three values of the pressure partition coefficient $\alpha$ have been considered in the computations: $1,0.1,0.01$. According to the criterion, regions in the plot where $(\alpha \xi / 2 g) \sin (\theta) \cos (\theta)$ exceeds 1 correspond to slopes of inclines on which self-fluidized motion of the density current may establish.
Analysis of fig. 4 suggests that for the smallest value of the pressure partition coefficient, $\alpha=0.01$, self-fluidization is never established, regardless of the value of $\theta$.

When the partition coefficient $\alpha$ is set at 0.1 , self-fluidization is established on inclines of slopes exceeding about $\theta_{c}=13^{\circ}$.

Self-fluidization is established at incline slopes of $\theta_{c}=2^{\circ}$ and larger when the upper limiting value of $\alpha$, namely 1 , is assumed in the computations.

The significance and implications of the selffluidization criterion are further clarified by fig. 5 . The speed $U$ of the density current is plotted in this figure as a function of the slope $\theta$. The speed $U$ is computed according to eqs. (3.2) or (3.3) depending on whether the flow is assumed to be in the self-fluidized state or not, respectively. Computations refer to arbitrary (though realistic) values of the depth of the flow $(h=5 \mathrm{~m})$ and of the angle of internal friction of the granular material $\left(\theta_{0}=27^{\circ}\right)$. The latter value corresponds to a coefficient of internal friction of the solids $\mu=0.5$. Again, plots corresponding to three values of the partition coefficient $\alpha$ (namely 1,0.1,0.01) are reported in the figure.

For $\alpha=0.01$, the flow is never self-fluidized, whatever $\theta$. Steady flow cannot establish on inclines whose slope is smaller than the

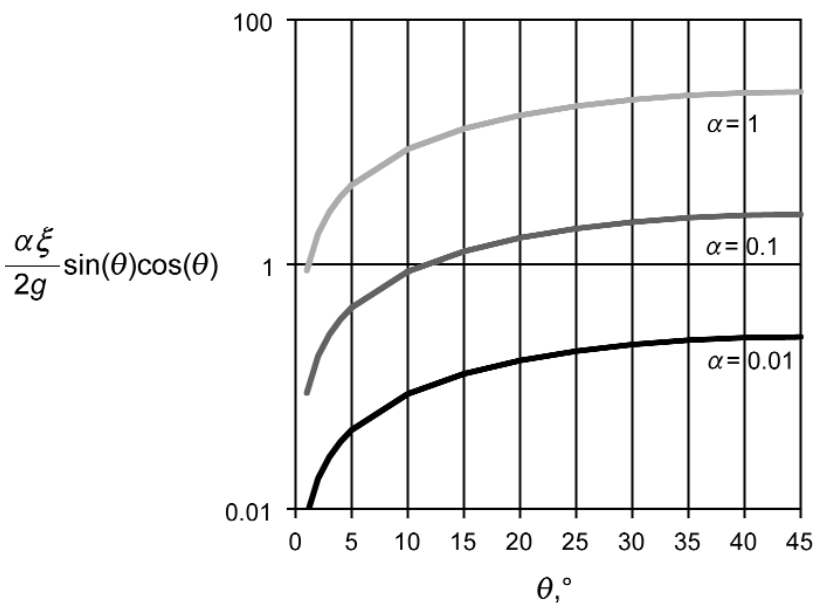

Fig. 4. Implementation of the self-fluidization criterion. 


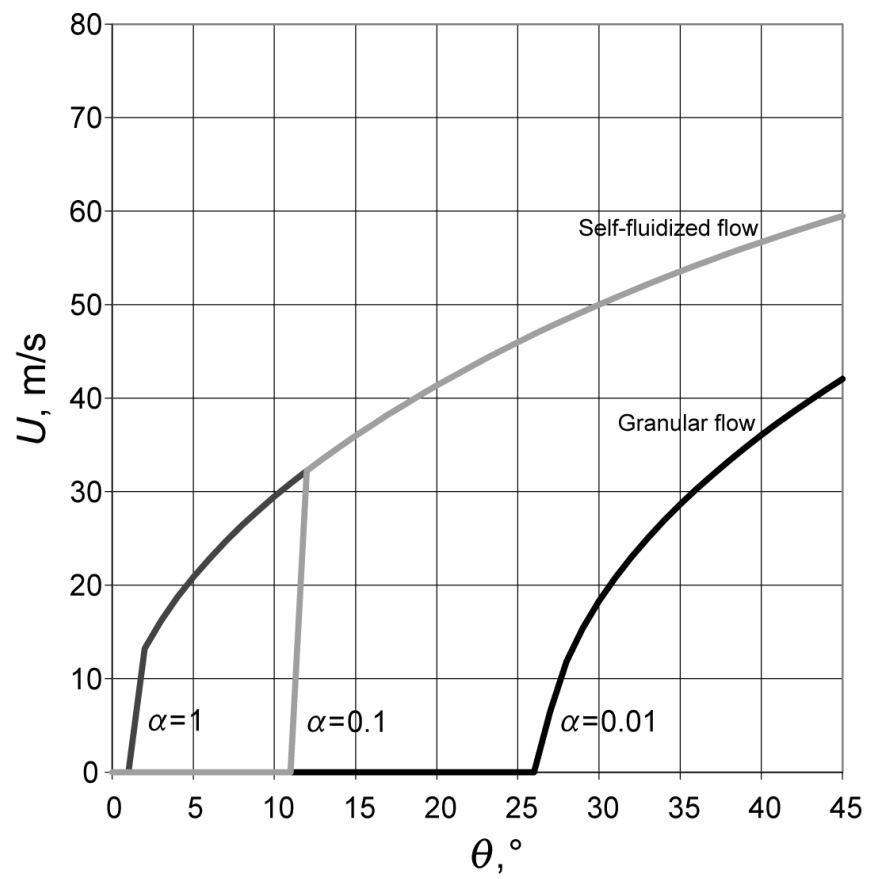

Fig. 5. Implementation of the criterion: computation of the front speed. Depth of the current: $h=5 \mathrm{~m}$; angle of internal friction $\theta_{0}=27^{\circ}$.

angle of internal friction $\theta_{0}(U=0)$. Steady granular flow establishes for $\theta>\theta_{0}$, at a speed $U$ computed according to eq. (3.3).

For $\alpha=0.1$ and $\alpha=1$, steady fluidized flow can be established at slopes larger than $\theta_{c}$ (where $\theta_{c}=13^{\circ}$ for $\alpha=0.1$ and $\theta_{c}=2^{\circ}$ for $\alpha=1$ ). The following flow regimes can be recognized, depending on the slope:

$\theta<\theta_{c}-$ Self-fluidization and steady flow of the current cannot be established. Regardless of the initial conditions, the asymptotic condition of the flow is the rest: $U=0$.

$\theta_{c}<\theta<\theta_{0}-$ Self-fluidization and steady flow of the density current can be established, provided that the initial conditions of the flow correspond to a fluidized state. The speed $U$ is represented, for any given slope, by the upper curve, corresponding to the self-fluidized state of the front. If the initial conditions of the flow correspond to a granular (non-fluidized) state, self-fluidization and steady motion of the current cannot establish and the flow comes to rest. $\theta>\theta_{0}-$ Steady motion of the current is established anyway. If the initial conditions of the flow correspond to a fluidized state, this state will be self-preserved by the motion of the current. The speed $U$ is given in this case by the upper curve in fig. 5 corresponding to the self-fluidized state. If the initial conditions of the flow correspond to granular (non-fluidized) flow, the flow will keep being granular. The speed $U$ is given by the lower curve corresponding to the granular state.

It is interesting to note that the critical slope $\theta_{c}$ plays the role of a bifurcation abscissa. Multiple steady states may establish for $\theta_{c}<\theta$ depending on the initial conditions of the flow. In particular it is noteworthy that steady flow of the density current can be established even at slopes smaller than the angle of internal friction $\theta_{0}$, provided that the initial conditions of the flow correspond to a fluidized state. This finding puts some emphasis on the proper choice of the initial conditions in experiments aiming at the characterization of unsteady granular flows along inclines. 
Similarly, these arguments shed some doubt on results obtained in experiments where the initial state of the flow was not properly documented (Takahashi and Tsujimoto, 2000).

The significance of the pressure partition coefficient $\alpha$ is better appreciated as one considers its physical meaning in the light of the rheological behaviour of the two-phase gas-solid system. The limiting case $\alpha=1$ corresponds to pseudohomogeneous two-phase flow (Wallis, 1969). This limiting case is characterized by the important feature that momentum transfer between the particulate phase and the interstitial gas phase is effective to the point that the slip velocity between the phases is negligible. On the other hand, $\alpha=0$ corresponds to the case where the stagnation pressure is entirely withstood by the granular solids. This corresponds to purely granular flow, with an expected strong influence of frictional stresses of Coulombian nature.

According to the criterion set by eq. (5.3), onset of self-fluidization is favoured by large values of $\alpha$, that is, as pseudo-homogeneous flow conditions are approached. On the other hand, establishment of pseudo-homogeneous flow is greatly enhanced by: a) fineness and lightness of the bed material; b) polydispersion of the bed material as regards particle size distribution, as momentum transfer from gas to coarser particles may occur, as in «dusty» gas, via the finer particles; c) sheared flow of the suspension, on account of the property of shear to stabilize homogeneous, i.e. bubble-free, fluidization of solids (Apicella et al., 1997). Features a) and b) are remarkable properties of pyroclastic granular materials. Maybe the recognition of the role of mutual momentum exchange between the particulate phase and the interstitial gas phase in the strongly accelerated flows at hand is the main outcome of the present study. It is recommended that parameters of the two-phase system which may affect interphase momentum exchange be carefully controlled in any experimental study aiming at the characterization of unsteady granular flows along inclines.

\section{Conclusions}

A simple criterion for the establishment of motion-induced self-fluidized flow of a pyroclas- tic density current is presented. It is based on a simplified formulation of the momentum balance equation on the frontal region of the dense flow, on the assessment of overpressures establishing in the basal shear layer of the flow as a consequence of solids fall-out, on the application of the criterion for fluidization of beds of granular solids. The criterion highlights the importance of the momentum exchange between the interstitial gas phase and the discrete particulate phase, expressed through a pressure partition coefficient $\alpha$. This parameter significantly affects the results of the application of the criterion. Its value depends on properties of the two-phase gas-solid system like particle size and density as well as polydispersity of the population of bed solids.

The application of the criterion is demonstrated. It is shown that steady self-fluidized motion of the density current can be established even on slopes smaller than the angle of internal friction of the granular solids - but larger than a critical slope $\theta_{c}$ which depends on the pressure partition coefficient - provided that the initial conditions of the flow correspond to the fluidized state. The critical slope $\theta_{c}$ corresponds to a bifurcation point, marking the limit beyond which multiple steady flow conditions, keeping memory of the initial conditions of the flow, may establish.

The proposed criterion is admittedly simple and its predictive capabilities have yet to be assessed. It might be helpful to shed light on some dynamical features of dense pyroclastic flows and to highlight aspects that need to be taken under control in experiments aiming at the characterization of dense granular flows.

\section{Acknowledgements}

The paper is part of an ongoing research program on «Dynamics of pyroclastic density currents and their impact on buildings», carried out under the umbrella of AMRA, Centro Regionale di Competenza sul Rischio Ambientale, Regione Campania. The author is grateful to Prof. Lucio Lirer, Prof. Antonio Marzocchella, Prof. Luciano Nunziante, Dr. Paola Petrosino, Dr. Teresita Gravina for useful discussion. The author is grateful to Augusto Neri and Olivier Roche for careful and constructive review of the paper. 


\begin{tabular}{|c|c|}
\hline \multicolumn{2}{|r|}{ Glossary } \\
\hline$B a$ & Bagnold number. \\
\hline$C_{f}$ & Coefficient in eqs. (2.2) and (2.3). \\
\hline$d$ & Particle diameter, $\mathrm{m}$. \\
\hline$f$ & Friction factor. \\
\hline$g$ & Acceleration due to gravity, $\mathrm{ms}^{-2}$. \\
\hline$h$ & Depth of the current, $\mathrm{m}$. \\
\hline$k$ & Coefficient in eq. (2.3), $\mathrm{kgm}^{-1}$ \\
\hline $\operatorname{Re}$ & Reynolds number. \\
\hline$R i$ & Richardson number. \\
\hline$U$ & Speed of the front, $\mathrm{ms}^{-1}$. \\
\hline$v_{z}$ & Particle velocity along the incline, $\mathrm{ms}^{-1}$. \\
\hline$y$ & Spatial coordinate orthogonal to the incline, $\mathrm{m}$ \\
\hline$z$ & Spatial coordinate along the incline, $\mathrm{m}$. \\
\hline$\Delta \rho=\rho_{m}-\rho_{a}$ & Difference between the average density of the current and the air density, $\mathrm{kgm}^{-3}$. \\
\hline$\eta$ & Effective viscosity of the gas-solid suspension, $\mathrm{Nsm}^{-2}$. \\
\hline$\eta_{g}$ & Gas viscosity, $\mathrm{Nsm}^{-2}$. \\
\hline$\theta$ & Slope of the incline, degree. \\
\hline$\theta_{c}$ & Critical slope according to the criterion eq. (5.3), degree. \\
\hline$\theta_{0}$ & Angle of internal friction of the particulate phase, degree. \\
\hline$\mu$ & Coefficient of internal friction of the particulate phase. \\
\hline$\xi$ & Constant in eq. (3.2), $\mathrm{m} \mathrm{s}^{-2}$. \\
\hline$\rho_{a}$ & Air density, $\mathrm{kg} \mathrm{m}^{-3}$. \\
\hline$\rho_{m}$ & Average density of the current, $\mathrm{kg} \mathrm{m}^{-3}$. \\
\hline$\rho_{p}$ & Particle density, $\mathrm{kg} \mathrm{m}^{-3}$ \\
\hline$\sigma_{y}$ & Normal stress in the particulate phase, $\mathrm{Pa}$. \\
\hline$\tau_{z y, f}, \tau_{z y, c}$ & Shear stress of frictional, collisional nature, respectively, $\mathrm{Pa}$. \\
\hline
\end{tabular}

\section{REFERENCES}

Apicella, E., M. D'Amore, G. Tardos and R. Mauri (1997): Onset of instability in sheared gas fluidized beds, AIChE J., 43, 1362-1365.

BAGNOLD, R.A. (1954): Experiments on a gravity-free dispersion of large solid spheres in a Newtonian fluid under shear, Proc. R. Soc., 225, 49-63.

BRITTER, R.E. and P.F. LINDEN (1979): The motion of the front of a gravity current travelling down an incline, $J$. Fluid. Mech., 99, 531-543.

Burgisser, A. and G.W. BERGANTZ (2002): Reconciling pyroclastic flow and surge: the multiphase physics of pyroclastic density currents, Earth Planet. Sci. Lett., 202, 405-418.

CAmpbell, C.S. (1990): Rapid granular flows, Ann. Rev. Fluid Mech., 22, 57-92.

DruitT, T. (1998): Pyroclastic density currents, in The Physics of Explosive Volcanic Eruptions, edited by J.S. Gilbert and R.S.J. Sparks, Geol. Soc. Spec. Publ. London, 145, 145-182.
Druitt, T., G. Bruni, P. Lettieri and J.G. Yates (2004): The fluidization behaviour of ignimbrite at high temperature and with mechanical agitation, Geophys. Res. Lett., 31 (2), L02604, doi: 10.1029/2003GL018593.

Esposti Ongaro, T., A. Neri, M. Todesco and G. MacedoNIO (2002): Pyroclastic flow hazard assessment at Vesuvius (Italy) by using numerical modeling, II. Analysis of flow variables, Bull. Volcanol., 64, 178-191.

Gravina, T., L. Lirer, A. Marzocchella, P. Petrosino and P. SALATINo (2004): Fluidization and attrition of pyroclastic granular solids, J. Volcanol. Geotherm. Res., 138, $27-42$.

HoPFINGER, E.J. (1983): Snow avalanche motion and related phenomena, Ann. Rev. Fluid Mech., 15, 47-76.

Levenspiel, O. (1998): Engineering Flow and Heat Exchange (Plenum Press, New York).

Marzocchella, A., P. Salatino, V. Di Pastena and V. LiRER (1998): Fluidization in pyroclastic flow, in Fluidization IX, edited by L.-S. FAN and T.M. KNOWLTON (Engineering Foundation, New York), 389-396.

Neri, A., A. Di Muro and M. Rosi (2002a): Mass partition 
during collapsing and transitional columns by using numerical simulations, J. Volcanol. Geotherm. Res., 115, 1-18.

Neri, A., P. Papale, D. Del Seppia and R. Santacroce (2002b): Coupled conduit and atmospheric dispersal dynamics of the A.D. 79 Plinian eruption of Vesuvius, J. Volcanol. Geotherm. Res., 120, 141-160.

Nunziante, L., M. Fraldi, L. Lirer, P. Petrosino, S. Scotellaro and C. Cicirelli (2003): Risk assessment of the impact of pyroclastic density currents on the towns located around Vesuvio: a non-linear structural inverse analysis, Bull. Volcanol., 65, 547-561.

Patton, J.S., C.E. Brennen and R.H. SABersky (1987): Shear flows of rapidly flowing granular materials, $J$. Appl. Mech., 54, 801-805.

Roche, O., M. Gilbertson, J. Phillips and S. Sparks (2002): Experiments on deaerating granular flows and implications for pyroclastic flow mobility, Geophys. Res. Lett., 29 (16), 1792, doi:10.1029/2002GL014819.

SAVAGE, S.B. (1979): Gravity flow of cohesionless granular materials in chutes and channels, J. Fluid Mech., 92, 53-96.

SPARKS, R.S.J. (1976): Grain size variations in ignimbrite and implication for the trasport of pyroclastic flows,
Sedimentology, 23, 147-188.

TAKAhashi, T. and H. Tsujimoto (2000): A mechanical model for Merapi-type pyroclastic flow, J. Volcanol. Geotherm. Res., 98, 91-115.

Todesco, M., A. Neri, T. Esposti Ongaro, P. Papale, G. Macedonio, R. Santacroce and A. Longo (2002): Pyroclastic flow hazard assessment at Vesuvius (Italy) by using numerical modeling, I. Large-scale dynamics, Bull. Volcanol., 64, 155-177.

VALENTINe, G.A. (1998): Damage to structures by pyroclastic flows and surges, inferred from nuclear weapons effects, J. Volcanol. Geotherm. Res., 87, 117-140.

Valentine, G.A. and K.H. Wohletz (1989): Numerical models of Plinian eruption columns and pyroclastic flows, J. Geophys. Res., 94, 1867-1887.

Wallis, G.B. (1969): One-dimensional Two-phase Flow (McGraw Hill)

WiLson, C.J.N. (1980): The role of fluidization in the emplacement of pyroclastic flow: an experimental approach, J. Volcanol. Geotherm. Res., 8, 231-249.

WiLSON, C.J.N. (1984): The role of fluidization in the emplacement of pyroclastic flow, 2. Experimental results and their interpretation, J. Volcanol. Geotherm. Res., 20, $55-84$. 